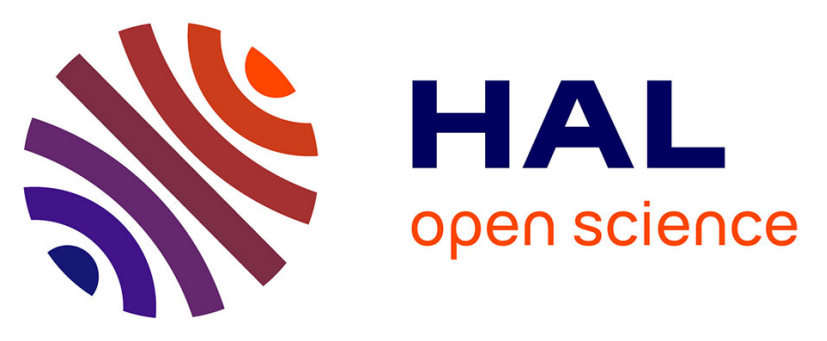

\title{
Millifluidic Synthesis of Polymer Core-Shell Micromechanical Particles: Toward Micromechanical Resonators for Acoustic Metamaterials
}

\author{
L. Lukyanova, L. Séon, A. Aradian, O. Mondain-Monval, J. Leng, R.
}

Wunenburger

\section{To cite this version:}

L. Lukyanova, L. Séon, A. Aradian, O. Mondain-Monval, J. Leng, et al.. Millifluidic Synthesis of Polymer Core-Shell Micromechanical Particles: Toward Micromechanical Resonators for Acoustic Metamaterials. Journal of Applied Polymer Science, 2013, 128 (6), pp.3512-3521. 10.1002/app.38411 . hal-00802535

\section{HAL Id: hal-00802535 \\ https://hal.science/hal-00802535}

Submitted on 6 Mar 2018

HAL is a multi-disciplinary open access archive for the deposit and dissemination of scientific research documents, whether they are published or not. The documents may come from teaching and research institutions in France or abroad, or from public or private research centers.
L'archive ouverte pluridisciplinaire HAL, est destinée au dépôt et à la diffusion de documents scientifiques de niveau recherche, publiés ou non, émanant des établissements d'enseignement et de recherche français ou étrangers, des laboratoires publics ou privés.

\section{(1)(1) $\$(0)$}

Distributed under a Creative Commons Attribution - NonCommercial - ShareAlikel 4.0 


\title{
Millifluidic Synthesis of Polymer Core-Shell Micromechanical Particles: Toward Micromechanical Resonators for Acoustic Metamaterials
}

\author{
L. Lukyanova, ${ }^{1,2}$ L. Séon, ${ }^{3,4,5}$ A. Aradian, ${ }^{1,2}$ O. Mondain-Monval, ${ }^{1,2}$ J. Leng, $^{3,4,5}$ R. Wunenburger ${ }^{6,7}$ \\ ${ }^{1}$ CNRS, CRPP, UPR 8641, F-33600 Pessac, France \\ ¿Université de Bordeaux, CRPP, UPR 8641, F-33600 Pessac, France \\ 3Université de Bordeaux, LOF, UMR 5258, F-33600 Pessac, France \\ ${ }^{4}$ CNRS, LOF, UMR 5258, F-33600 Pessac, France \\ ${ }^{5}$ RHODIA, LOF, UMR 5258, F-33600 Pessac, France \\ EUniversité de Bordeaux, LOMA, UMR 5798, F-33400 Talence, France \\ ${ }^{7}$ CNRS, LOMA, UMR 5798, F-33400 Talence, France \\ Correspondence to: R. Wunenburger (E-mail: regis.wunenburger@u-bordeaux1.fr)
}

\begin{abstract}
We present two routes of millifluidic based synthesis of solid core solid shell particles with submillimeter size. These particles, which behave as mechanical resonators, are key components of targeted three dimensional locally resonant acoustic meta materials working in the ultrasonic frequency range. The first route involves the encapsulation of a solid core into a liquid shell, which is then in line solidified through gelation. The second route involves two successive coflows followed by two solidifications triggered by UV irradiation and complexation, respectively. Original solutions to several practical issues are presented. The advantages and drawbacks of both routes are analyzed in the light of the targeted specifications of the resonators.
\end{abstract}

KEYWORDS: composites; microfluidics; gels

\section{INTRODUCTION}

In 1968, Veselago ${ }^{1}$ theoretically explored the physics of electromag netic (em) propagation in materials having both their dielectric permittivity $\varepsilon$ and magnetic permeability $\mu$ negative. His theoreti cal work remained speculative until it was shown that simultaneous negative effective values of $\varepsilon$ and $\mu$ can be achieved in propagating media containing a sufficient concentration of physical entities that are able to resonate with the incoming em field, thus leading to negative values of the effective optical refrac tive index close to the resonance frequencies of the resonators. ${ }^{2}$ The synthesis and characterization of such materials called locally resonant metamaterials have motivated intensive worldwide research $^{3}$ due to the large number of potential applications of em metamaterials (super lenses, cloaking materials, subwavelength optical microscopy, filters, antenna, wave guides, etc.). Realizing that several of these promising applications could be transposed to acoustics, ${ }^{4}$ researchers have recently begun to work on the design and synthesis of materials that would exhibit negative values of the effective density $\rho_{\text {eff }}$ and/or of the effective compressibility $\kappa_{\text {eff- }}$. Such acoustic metamaterials indeed open the route to many appli cations such as planar perfect acoustic lenses, ${ }^{5,6}$ acoustic cloaking devices, ${ }^{7}$ subwavelength acoustic microscopy, ${ }^{8}$ frequency selective acoustic isolators and attenuators, ${ }^{9,10}$ and waveguides. The homogenization process leading to the achievement of effective acoustic properties for a composite material requires the acoustic wavelength to be much larger than the material microstructure. The higher the targeted working frequency, the smaller the wave length and consequently the smaller the resonators have to be. Consequently, while negative effective densities and/or compressi bilities could be obtained in the audible frequency range for in the case of one, ${ }^{11,12}$ two, and three dimensional (3D) propagation ${ }^{9,13}$ using macroscopic and hand made resonators, similar effects could be obtained in the ultrasonic range only in one ${ }^{11}$ and two dimensions, ${ }^{6,7,14}$ due to the difficulty to manually fabricate and assemble submillimetric resonators. Pushing the working fre quency of 3D LRAM up to ultrasonic frequencies requires to downsize the resonators down to the submillimeter range. This represents several design and fabrication issues.

Among the variety of resonators theoretically proposed for achieving locally resonant acoustic metamaterials (LRAM), hard 
core soft shell resonators, which have been proposed in Ref. 15 as a mean to obtain negative effective densities, have a particu larly simple structure. Moreover, it has been experimentally shown in Ref. 9 that negative effective densities could actually be obtained around $400 \mathrm{~Hz}$ in a 3D LRAM composed of hand made centimeter sized hard lead spheres embedded in a soft polymer shell and further integrated into a hard matrix. This kind of resonators thus constitutes an attractive design for $3 \mathrm{D}$ LRAM working in the ultrasonic range. Interestingly, such sizes lie within the scope of fabrication techniques such as microflui dics. The scope of this work is to present in detail several proc esses of the synthesis of submillimetric acoustic resonators using millifluidic techniques.

Our work is organized as follows. In Materials Design section, we present the main results of the design study of the LRAM based on the model given in Ref. 15. In Millifluidic Fabrication of the Resonators section, we present two routes of synthesis of core shell resonators based on millifluidics. We study the dependence of the dimensions of the core shell particles on the experimental conditions, and we highlight the original features of the implemented millifluidic processes. Finally, we compare both routes in terms of performances and versatility.

\section{MATERIALS DESIGN}

The geometry of the mechanical resonator proposed in Ref. 15 is shown in Figure 1(a). When submitted to the acceleration associated to an incident, monochromatic, plane acoustic wave, the hard core of the resonator oscillates within the soft shell parallel to the direction of the wave, behaving like a harmonic oscillator whose stiffness is provided by the resistance to shear of the shell and whose inertia is mainly provided by the mass of the core. The exact mechanical response of a hard core soft shell single spherical particle suspended in a hard matrix to long wavelength acoustic waves has been modeled in Ref. 15 in the limit of infinitely rigid core and matrix. We used this ana lytical model to design the core shell resonators. We also numerically studied the role of viscous dissipation on the reso nance strength as function of the dimensions of the resonator. Although the details of this study will be published elsewhere, we give below the main outcomes of the model concerning the resonance behavior.

Noting $a$ the core radius, $b$ the shell outer radius, $\rho_{3}$ (resp. $\rho_{2}$ ) the density of the material constituting the core (resp. the shell), $\lambda_{2}$ the first Lamé coefficient of the material constituting the shell, and $G_{2}^{\prime}$ its second Lamé coefficient, that is, the elastic component of its (complex) shear modulus $G_{2}\left(=G_{2}^{\prime}+i G_{2}^{\prime \prime}{ }_{2}\right)$, the resonance frequency $f_{\text {res }}$ of the harmonic motion of the core within the shell approximately satisfies the following scaling law:

$$
f_{\text {res }} \sim \sqrt{\frac{G_{2}^{\prime}}{\rho_{3} a^{2}}}
$$

The increase of $f_{\text {res }}$ with decreasing $a$ justifies our efforts of miniaturization of the resonator size. A typical frequency dependence of the effective density $\rho_{\text {eff }}$ of a suspension of such
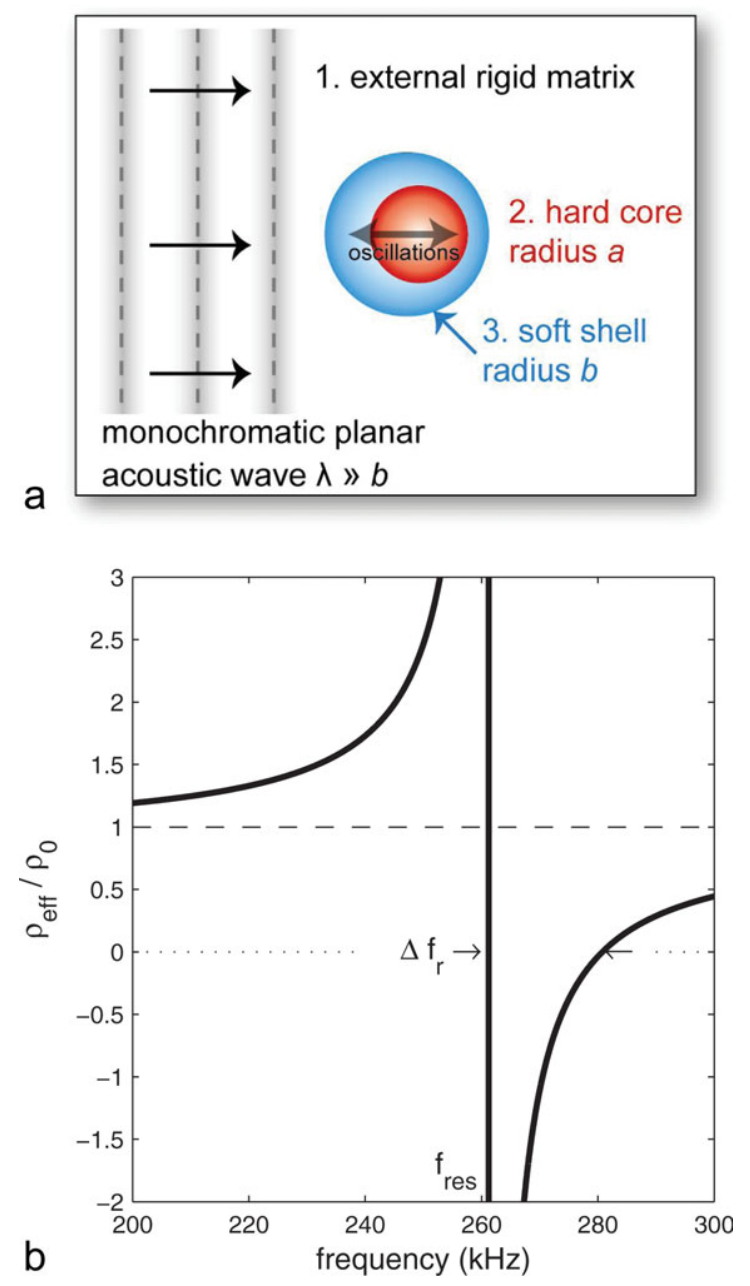

Figure 1. (a) Schematic representation of the hard core soft shell resonator. (b) Predicted variation of the dimensionless effective density $\rho_{\text {eff }} / \rho_{0}$ of the suspension of core shell resonators as function of the frequency $f$ of the acoustic wave. $f_{\text {res }}$ is the resonance frequency and $\Delta f_{r}$ the frequency band in which the effective density of the suspension is predicted to be negative $\left(\rho_{1} \quad 1 \mathrm{~g} \mathrm{~cm}^{-3}, \quad \rho_{2} \quad 1.09 \mathrm{~g} \mathrm{~cm}^{-3}, \quad \rho_{3} \quad 8.86 \mathrm{~g} \mathrm{~cm}^{-3}, \quad G_{2}^{\prime} \quad 0.6 \mathrm{MPa}\right.$, $\lambda_{2} \quad 3.6, \mathrm{GPa}, a \quad 50 \mu \mathrm{m}$, and $b \quad 75 \mu \mathrm{m}$ volume fraction of resonators in the material: $\phi \quad 0.1)$. [Color figure can be viewed in the online issue, which is available at wileyonlinelibrary.com.]

mechanical resonators divided by its static value $\rho_{0}$ is shown in Figure 1(b), where we indeed note the occurrence of a fre quency band $\Delta f_{r}$ over which $\rho_{\text {eff }}$ is predicted to be negative. For practical reasons, $\Delta f_{r} / f_{\text {res }}$ has to be as broad as possible in order to compensate, for instance, for the possible variability of the resonator mechanical properties and dimensions but also, and importantly, for dissipation. Numerically, we found that $\Delta f_{r} / f_{\text {res }}$ increases with $\left(\begin{array}{ll}b & a\end{array}\right) / a$ up to a plateau for ( $b \quad a) / a<0.5$. The shell thickness thus shall be reduced down to $0.5 a$, below which there is no real subsequent gain in terms of band width. We also found that $\Delta f_{r} / f_{\text {res }}$ strongly depends on $\rho_{3} \quad \rho_{2}$ and almost vanishes for $\rho_{3}=\rho_{2}$. Although the density matching between the core and the shell does not suppress the resonance, it makes it experimentally unattainable $\left(\Delta f_{r} / f_{\text {res }} \rightarrow 0\right)$. 


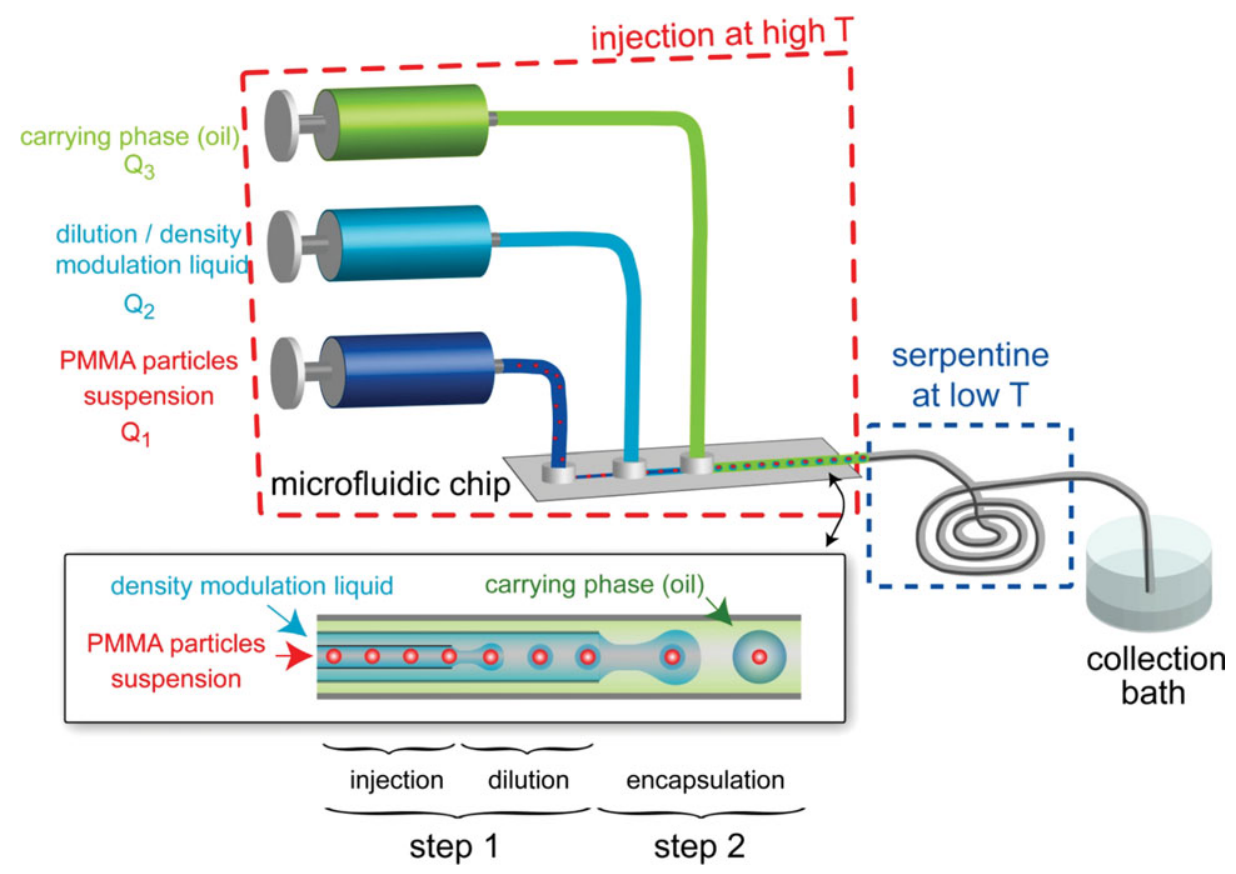

Figure 2. Schematic representation of the millifluidic chip implemented for producing core shell objects using the combination of Method 1 for Step 1 and Method 1 for Step 2 (C1 E1 in the text): a density matched suspension of solid polymer particles is injected at high temperature (Step 1, dark blue syringe), density modulated by dilution (light blue syringe), and then encapsulated (Step 2, green syringe) into agarose droplets, which are eventually gelled at low temperature in the serpentine device. [Color figure can be viewed in the online issue, which is available at wileyonlinelibrary.com.]

\section{MILLIFLUIDIC FABRICATION OF THE RESONATORS}

We now present the different experimental routes that we explored for achieving the synthesis of the resonators. In this work, we understand the process of fabrication of core shell particles as composed of two different steps that will constitute two fabrica tion modules. As we describe now, this approach allows for the development of a large variety of objects. The two steps that are required for the fabrication of our objects are the following ones:

Step 1: Fabrication of the hard core of the microresonator. Step 2: Millifluidic encapsulation of the core into a soft shell.

A third step is also required to build the final LRAM that is the incorporation of a controlled amount of the core shell particles into a rigid matrix.

For achieving Step 1, we have investigated two different meth ods that are as follows:

C1: The use of colloidal particles that are in a solid state "right from the start" and that will be encapsulated as such into the material that will later constitute the soft shell.

C2: The use of an initially liquid phase that will be first proc essed into spheres of controlled size within the microchan nel, then solidified (in our case through inline UV polymerization reaction), and finally integrated as such into the material that will later constitute the soft shell.

For Step 2, we have also developed two possible experimental methods that are as follows:

E1: The use of an aqueous physical hydrogel whose gelation occurs through an inline temperature quench.
E2: The use of an aqueous physical gel whose gelation occurs through the inline triggering of a complexation reaction.

Encapsulation has been largely described in literature. For cap sules with size in the $100 \mu \mathrm{m}$ range, it mostly relies on micro fluidic liquid liquid processes and exploits the spontaneous emulsification due to the contact of immiscible fluids in con fined geometries. ${ }^{16}$ A large set of geometries and devices have been produced, and we use the seminal work of Ref. 17 that demonstrated the use of nested capillaries for the generation of calibrated multiple emulsions.

The subsequent polymerization exploits most often photocura ble polymers or hydrogel curable; thanks to a sol gel process. A review can be found in Ref. 18. The advantages of using an aqueous gel for constituting the shell are that (i) its viscoelastic properties can be continuously tuned by adjusting its composi tion and (ii) its shear modulus is generally much smaller than those of organic polymers, which have been used for constitut ing the core of the resonators, a condition of validity of the model of Ref. 15.

The different experimental processes that will be described for each step of fabrication might be developed as independent and connectable modules that can lead to the formation of a large variety of objects when combined in four different ways such as C1 E1, C1 E2, C2 E1, or C2 E2. In the following, we describe the build up of the different modules and the characteristic fea tures of the obtained materials. In this work, we have chosen to implement two combinations among the four possible, namely C1 E1 and C2 E2. 


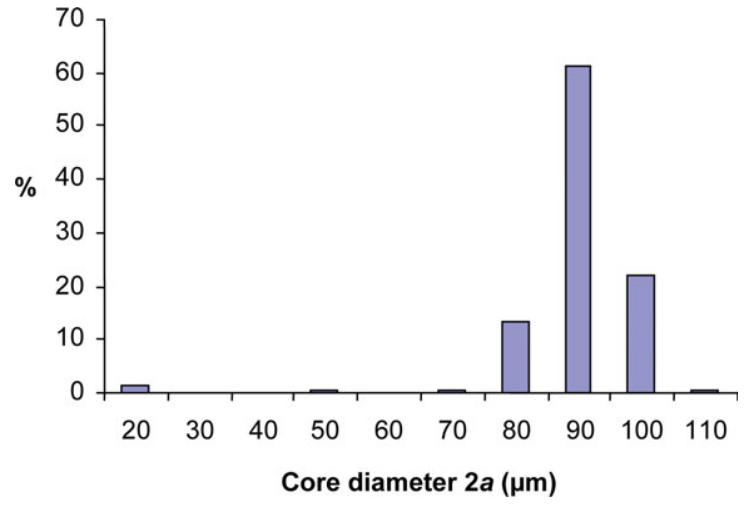

Figure 3. Histogram of the diameters of PMMA particles. [Color figure can be viewed in the online issue, which is available at wileyonlinelibrary.com.]

\section{Combination C1 E1}

First, we describe the implementation of a combination of an initially solid particle as the core and of a physical hydrogel as the shell, whose gelation is controlled by temperature.

Core Fabrication Step. As discussed in Materials Design sec tion, the width of frequency band over which the effective den sity of the targeted LRAM is predicted to be negative is highly dependent on the density contrast between the core and the shell. This imposes severe constraints on the choice of the core material that should be as dense as possible. On the other hand, manipulating fluids containing high density colloidal particles in capillary tubes is a complicated procedure due to the rapid sedimentation of the particles within the microchannel. To cir cumvent this difficulty, we have used the millifluidic setup sche matized in Figure 2.

The solid colloidal particles are first dispersed in a density matched liquid mixture of water and glycerine principally in order to ease their injection into the channels by suppressing sed imentation. Then the density of the suspending fluid is decreased through its controlled mixing with another fluid having a density different from the one of the suspension (see Figure 2). To avoid the sedimentation of the particles downstream of the mixing stage, this dilution stage has to be as close as possible to the encapsulation stage. In the case that we discuss here, the use of a pure agarose/water solution led to lower the density of the sus pending fluid below one of the particles, but this step can also be used to increase the density of the suspending fluid well above the density of the particles by using, for example, heavy salt solu tions such as $\mathrm{Pb}$ or $\mathrm{Au}$ ions dispersed in water.

We have used poly(methylmethacrylate) (PMMA) particles from Atoglass as the solid core of the resonator. The initial particles are available as a powder of particles of diameters comprised between 10 and $500 \mu \mathrm{m}$. To reduce its size dispersity, the pow der was first dried in an oven at a very low hygrometry level in order to remove any trace of moisture and then sieved using metallic grids of appropriate mesh sizes. As shown in Figure 3, the diameter of more than $95 \%$ of the sieved PMMA particles is $100( \pm 10) \mu \mathrm{m}$. The PMMA particle density is $\rho=1.19 \mathrm{~g} / \mathrm{cm}^{3}$, which determines the required value of the suspending fluid density, hence the proportions of water and glycerine. As the next step involves the formation of a shell made of an agarose hydrogel, the suspending fluid also contains agarose at a con centration ranging from 0.5 to 3 wt \%. As justified after, in order to keep the agarose/glycerine/water mixture in a liquid state, the temperature $T$ of the suspension was first increased above $T_{\text {fluid, }}$ as defined in Figure 4 , and then slowly cooled down to $50^{\circ} \mathrm{C}$, then injected into the millifluidic circuit, which was also kept at this temperature. A combination of surfactants [sodium dodecyl sulfate (SDS) and nonionic Pluronic F127 or Tween 80] had to be added to the solution in order to suppress the aggregation of PMMA particles at high temperature due to the good to bad solvent transition of the particles surface poly mer chains in the solution. A typical composition of the aga rose/glycerine/SDS/F127/water mixture is in wt \%: 1/74/0.2/1/ 23.8 in which 0.35 wt $\%$ of PMMA particles was dispersed. The dilution stage involves the mixing of the suspension (flow rate $Q_{1}$ ) with a second solution of 1 wt $\%$ agarose in pure water
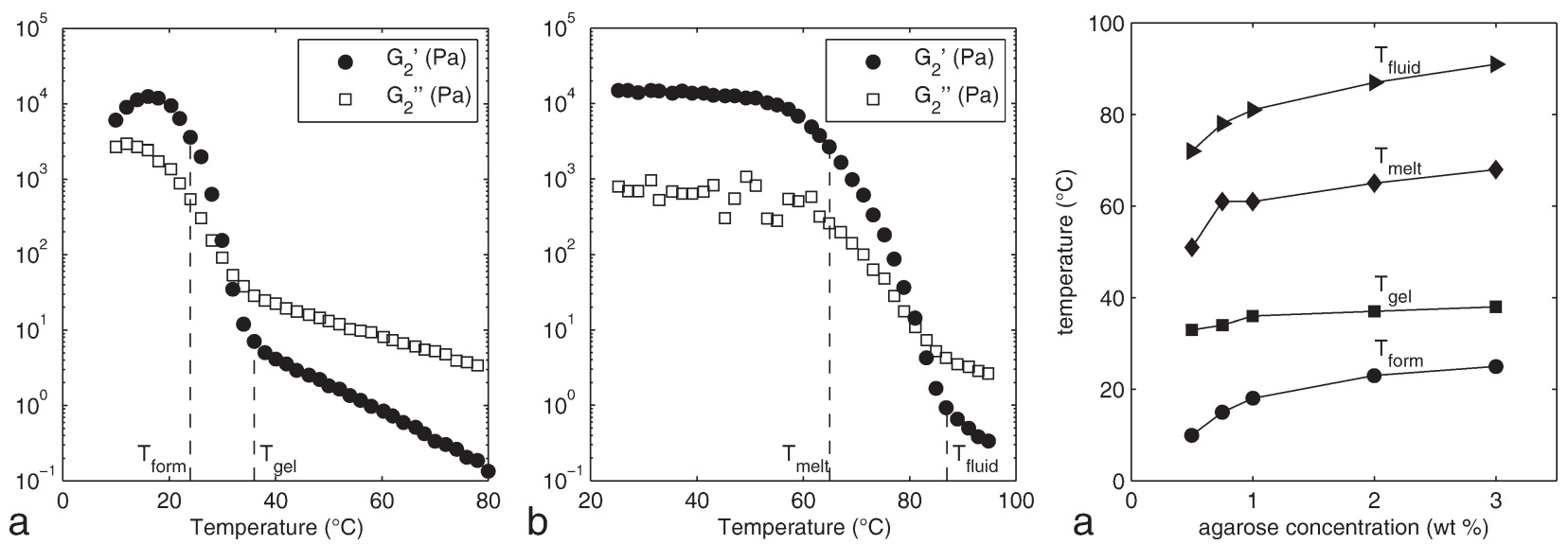

Figure 4. (a) Temperature dependence of the complex elastic modulus $G_{2} \quad G_{2}^{\prime}+i G^{\prime \prime}{ }_{2}$ of a 1 mm thick sample of a 2 wt $\%$ agarose/74 wt $\%$ glycer ine/2 wt \% Tween $/ 0.2$ wt \% SDS $/ 21.8$ wt \% water mixture during (a) cooling at a rate of $1{ }^{\circ} \mathrm{C} / \mathrm{min}$, (b) heating at $1^{\circ} \mathrm{C} / \mathrm{min}$, and (c) dependence of the temperatures of beginning $T_{\text {gel }}$ and achievement $T_{\text {form }}$ of solidification and of beginning $T_{\text {melt }}$ and achievement $T_{\text {fluid }}$ of melting of $X$ wt $\%$ agarose/74 wt $\%$ glycerine/2 wt $\%$ Tween $/ 0.2$ wt $\%$ SDS/ $(23.8 \quad X)$ wt \% water mixtures versus agarose concentration $X$. 
with a density of $1 \mathrm{~g} / \mathrm{cm}^{3}$ (flow rate $Q_{2}$ ), as sketched in Figure 2. The millifluidic setup used for achieving the dilution, sche matized in Figure 2, was fabricated using nested glass capillary tubes of round and square cross sections in order to ensure self centering. ${ }^{17}$ Such technology indeed permits to generate multilevel coflows with an axial symmetry. The glass capillary tubes from Vitrocom used for the suspension injection and the dilution stage are made of borosilicate and had the following dimensions: inner diameter ID $=150 \mu \mathrm{m}$ and outer diameter $\mathrm{OD}=250 \mu \mathrm{m}$ and $\mathrm{ID}=300 \mu \mathrm{m}$ and $\mathrm{OD}=400 \mu \mathrm{m}$. The resulting density contrast between the particles and the suspend ing fluid achieved after the dilution stage, estimated from the densities of the two mixed solutions by assuming linearity upon dilution with known flow rates, is $0.1 \mathrm{~g} / \mathrm{cm}^{3}$.

To determine the temperatures of the high temperature and low temperature stages of the millifluidic circuit, a study of the solidi fication and melting of agarose/glycerine/surfactants/water mix tures as function of agarose concentration has been performed. Solidification (resp. melting) experiments of $1 \mathrm{~mm}$ thick samples have been conducted by ramping up (resp. down) the samples temperature at a slow variation rate of $+1^{\circ} \mathrm{C} / \mathrm{min}\left(\right.$ resp. $1^{\circ} \mathrm{C} /$ min) and by monitoring their complex elastic modulus at low frequency $(0.1 \mathrm{~Hz})$ and for small relative deformation (1\% strain) using a temperature regulated Texas Instrument AR 1000 rheom eter. The complex elastic modulus $G_{2}$ of the studied mixtures exhibits large hysteresis upon melting and solidification. The var iations of this complex elastic modulus of a $2 \mathrm{wt} \%$ agarose/74 $\mathrm{wt}$ $\%$ glycerine $/ 2$ wt $\%$ Tween/0.2 wt \% SDS/21.8 wt \% water mix ture versus temperature during the up/down temperature ramps, shown in Figure $4(\mathrm{a}, \mathrm{b})$, indicate that its solidification indeed begins at temperature $T_{\text {gel }}$ and is achieved at $T_{\text {form }}$, while its melt ing begins at $T_{\text {melt }}$ and is achieved at $T_{\text {fluid, }}$ which strongly differ from $T_{\text {gel }}$ and $T_{\text {form }}$, respectively. We have checked that during the temperature ramps, the evolution of the samples was actually quasi static. The variations of $T_{\text {gel, }}, T_{\text {form }}, T_{\text {melt }}$, and $T_{\text {fluid }}$ as func tion of agarose concentration, shown in Figure 4(c), indicate that, for the investigated range of agarose concentration, injection and encapsulation have to be performed at a temperature larger than $T_{\text {gel, }}$ while complete solidification of the shells is achieved by quenching the core shell particles below $10^{\circ} \mathrm{C}$. This is why a refri gerated serpentine was added downstream of the encapsulation stage in order to ensure a $10 \mathrm{~min}$ stay of the core shell particles at $10^{\circ} \mathrm{C}$ before their collection.

Shell Fabrication Step. We note that the microfluidic encapsu lation of solid particles is rarely described in literature. We found that encapsulation of solid particles mainly concerns nano to microparticles (quantum dots, latexes, etc.), ${ }^{19,20}$ while larger objects are restricted to cells ${ }^{21}$ and soft particles. ${ }^{22}$ This positions our work as an original way of encapsulating solid, non Brownian particles using millifluidics.

The particle encapsulation step was then performed using an im miscible carrier fluid (Silicone oil, with viscosity $110 \mathrm{mPa} \mathrm{s}$ ), called the outer fluid hereafter, flowing through a (ID $=500 \mu \mathrm{m}$ and $\mathrm{OD}=700 \mu \mathrm{m}$ ) borosilicate capillary tube (see Figure 2). The number of PMMA particles per capsule depends on both the particles concentration in the suspension on its flow rate $Q_{1}+$

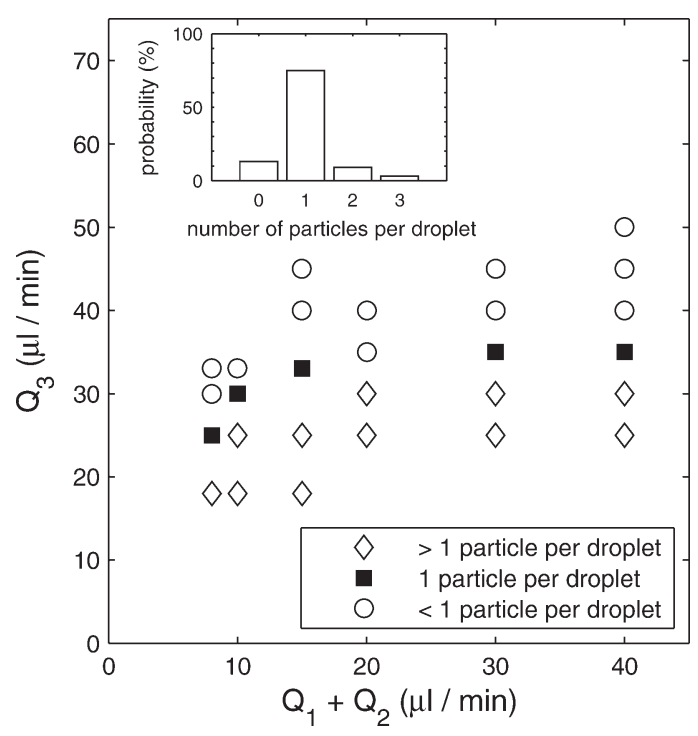

Figure 5. (a) Flow diagram of the encapsulation stage of the $\mathrm{C} 1 \mathrm{E} 1$ route in the drop only region as a function of internal $\left(Q_{1}+Q_{2}\right)$ and external $\left(Q_{3}\right)$ flow rates (the concentration of PMMA particles in the suspension is 0.35 wt $\%$ upstream from the density modulation stage). (b) Histogram showing the percentage (\%) of objects having a given number of encapsu lated PMMA particles in the operational conditions $\left(Q_{1}+Q_{2} \quad 30 \mu \mathrm{L} /\right.$ $\min$ and $\left.Q_{3} \quad 35 \mu \mathrm{L} / \mathrm{min}\right)$.

$Q_{2}$ and on the outer fluid flow rate $Q_{3}$. Figure 5(a) displays the dynamic flow diagram of the encapsulation process. We focused on the region of the flow diagram where drops are observed, thus disregarding the possible drop to jet transition. ${ }^{16}$ In this drop only region, the number of PMMA particles in the final capsule strongly depends on the operational parameters ${ }^{23}$; having fixed the geometrical parameters of our device, we studied the encap sulation conditions as a function of the flow rates and the con centration of particles in the suspension. Capsules containing on average a single PMMA particle are obtained only in a restricted domain of the flow diagram. In this domain, the typical percent age of capsules containing only one particle is $80 \%$, as shown in Figure 5(b). Moreover, the encapsulation statistics is known to follow a Poisson law, ${ }^{23}$ compatible with the observed distribution.

We thus fabricated continuously gelled core shell particle at a moderate throughput (order of 110 drops/s) for several hours. Figure 6(a) displays a SEM picture of the obtained core shell par ticles. These objects were collected, rinsed, and then dispersed in a UV polymerizable mercapto ester based monomer adhesive (Norland Optical Adhesive 65). Using this fabrication process, we managed to fabricate a thin circular slab (thickness $5 \mathrm{~mm}$ and radius $5 \mathrm{~cm}$ ) loaded with about $10 \%$ (vol) with the core shell particles and indeed observed that the particles kept their integ rity. Pictures of the final dispersion are shown in Figure 6(b,c).

\section{Combination C2 E2}

We now describe the implementation of a combination of an initially liquid core that is polymerized in situ and of a shell whose gelation is triggered by a chemical reaction. 

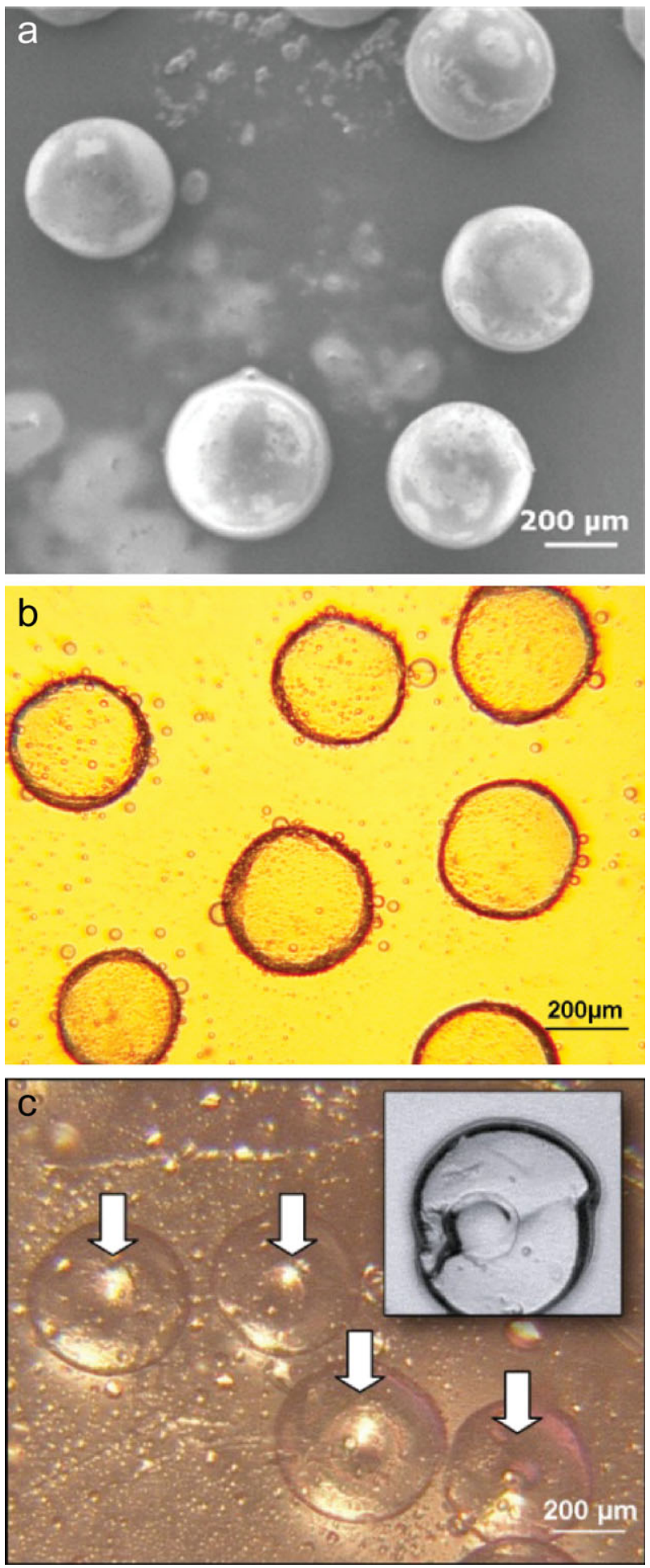

Figure 6. (a) SEM picture (size $1.25 \times 1.05 \mathrm{~mm}^{2}$ ) of the capsules whose core is an initially solid PMMA and whose shell is made of agarose/water gel (route 1). (b) Transmission optical microscopy picture of the same core shell particles embedded in the solid polymer matrix. (c) Reflec tion optical microscopy picture of the same core shell particles in the solid matrix. The white arrows point to the encapsulated PMMA particles (insert up right: optical microscopy picture of a crushed object showing the inserted particle (same scale as main picture). [Color figure can be viewed in the online issue, which is available at wileyonlinelibrary.com.]
Core Fabrication Step. The monomer that we used to form the core of the resonator is ethylene glycol dimethacrylate. Its polymerization is triggered by UV irradiation in the presence of 2 hydroxy 2 methylpropiophenone $(2 \mathrm{H} 2 \mathrm{M})$ that is the polymer ization initiator. EGDA possesses two double bonds and the reaction that occurs is a radical polymerization.

In this route too, we used glass capillary tubes to promote the formation of EGDA droplets. However, to avoid any preferential wetting of the glass tube by the EGDA solution, the tubes first had to be surface treated with a solution of hydroxyethylcellu lose (HEC), which is a well established way of increasing the hydrophilicity of a surface. The droplet formation stage is made of an untreated inner capillary tube with circular cross section and dimensions $(\mathrm{ID}=150 \mu \mathrm{m}$ and $\mathrm{OD}=250 \mu \mathrm{m})$ centered in an intermediate, HEC treated capillary tube with square cross section, and dimensions (ID $=300 \mu \mathrm{m}$ and $\mathrm{OD}=400 \mu \mathrm{m}$; see Figure 7). The solution injected in the inner capillary tube is composed of $5 \mathrm{wt} \%$ of the initiator $2 \mathrm{H} 2 \mathrm{M}$ dispersed in EGDA (flow rate $Q_{1}$ ). The solution injected in the intermediate capil lary tube, which will further constitute the shell of the final objects, is a $1 \mathrm{wt} \%$ solution of alginate in water (flow rate $Q_{2}$ ). First, we measured the dependence of the diameter $2 a$ of EGDA droplets on the ratio of the external to internal flow rates $Q_{2} / Q_{1}$. As shown in Figure 8, the best power law fit of these variations is $2 a \propto\left(Q_{2} / Q_{1}\right)^{-0.14}$, in good agreement with the empirical law evidenced in Ref. 24. Experimentally, droplets with diameter ranging from 180 to $280 \mu \mathrm{m}$ could be produced. After their formation, the droplets were submitted to a UV illu mination (DYMAX Blue wave 200 lamp) triggering the on fly polymerization while still in the HEC treated glass capillary tube. We observed that, while indeed triggering the polymeriza tion of the EGDA droplets, UV irradiation also seriously dam aged the HEC layer adsorbed at the surface of the tube, as it can be observed in Figure 9. As a consequence, EGDA droplets had to be first encapsulated and then transferred into an untreated tube before their UV irradiation.

Shell Fabrication Step. Alginate, which is a mixture of several types of polysaccharides from Sigma Aldrich, was chosen as polymer for constituting the shell of the resonators. When dis solved in water, alginate solution can be turned into a physical gel by complexation with $\mathrm{Ca}^{2+}$ ions $(\mathrm{CaCl}$ monohydrate from Fisher). Because the encapsulation of EGDA droplets into algi nate has to be performed before its gelation, we chose to directly use the external phase carrying the core shell particles as $\mathrm{Ca}^{2+}$ ions provider. Oleic acid, which simultaneously exhibits poor solubility in alginate and sufficiently dissolves $\mathrm{Ca}^{2+}$ ions, was chosen as external phase. Calcium ions were indirectly dis solved into oleic acid by first dissolving $\mathrm{CaCl}_{2}$ into butanol, then by adding and mixing oleic acid to this solution (the two phases being miscible), and eventually evaporating butanol, resulting in what we further refer to as "salted" oleic acid. ${ }^{25}$

To avoid the formation of plugs of alginate gel immediately after starting the flows, that is, before attaining steady state con ditions for the encapsulation process, a mixing module (mag netic millimixer shown at the top of Figure 7) was added upstream from the encapsulation stage. This module allowed 

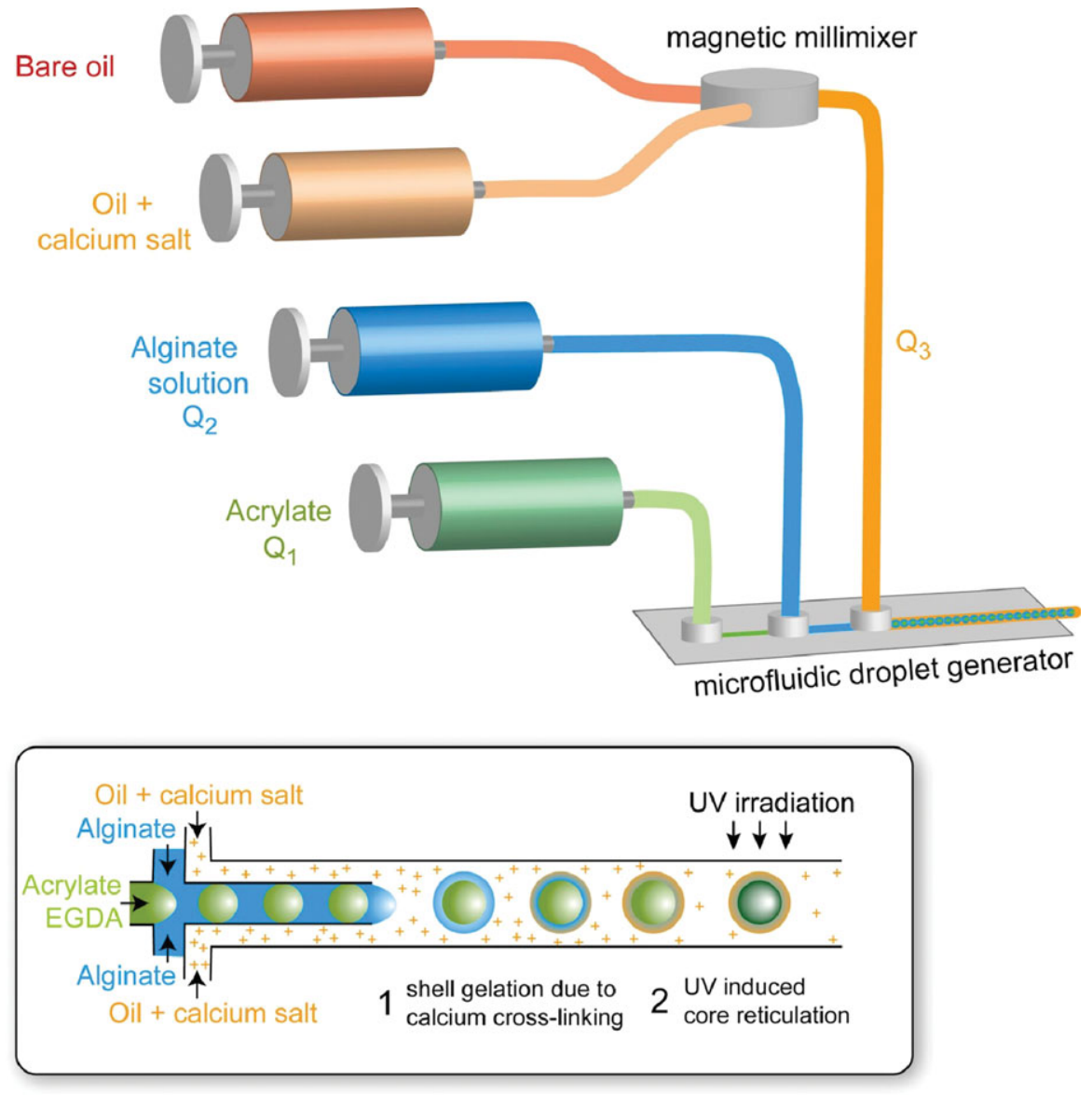

Figure 7. Schematic presentation of the millifluidic chip implemented for producing core shell objects using the combination of Method 2 for Step 1 and Method 2 for Step 2 (C2 E2 in the text): liquid EGDMA monomers and 2H2M initiator are injected (dark green syringe) and form droplets in the alginate solution (Step 1, blue syringe). The encapsulation step is achieved by the further injection of the resulting two phase system into an oil/calcium salt solution (Step 2) that is obtained through the mixing of a pure (dark brown syringe) and a salted oleic solution (light brown syringe) in the magnetic millimixer. The gelation of the shell occurs owing to the diffusion of $\mathrm{Ca}^{2+}$ ions through the core made of alginate solution. The polymerization of the core is triggered by UV irradiation downstream from the encapsulation stage. [Color figure can be viewed in the online issue, which is available at wileyonlinelibrary.com.]

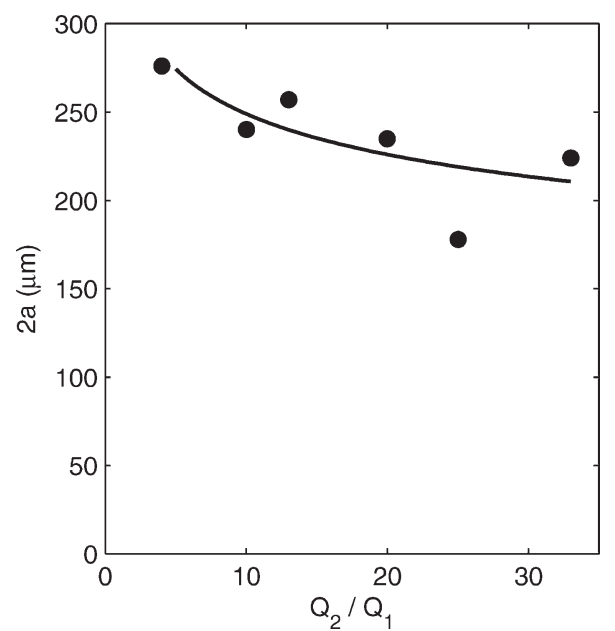

Figure 8. Variation of the diameter $2 a$ of EGDA droplets (core) as a function of the ratio of the flow rates in the outer $\left(Q_{2}\right)$ and the inner $\left(Q_{1}\right)$ channels of the core formation stage. The solid curve is the best fit of the data set to a power law, leading to $2 a \sim\left(Q_{2} / Q_{1}\right)^{0.14}$. for a progressive increase in the salinity of the salted oleic acid injected into the encapsulation stage once a steady regime of encapsulation is attained by tuning the flow rates of pure oleic acid and of the salted oleic acid injected into the module.

The gelation kinetics of $1 \mathrm{wt} \%$ alginate in water was measured as a function of $\mathrm{Ca}^{2+}$ concentration by evaluating the time de pendence of the amplitude of the Brownian motion of $1.1 \mu \mathrm{m}$ diameter fluorescent latex beads (Invitrogen) suspended in $1 \mathrm{~mm}^{3}$ typical volume droplets of alginate solution injected in

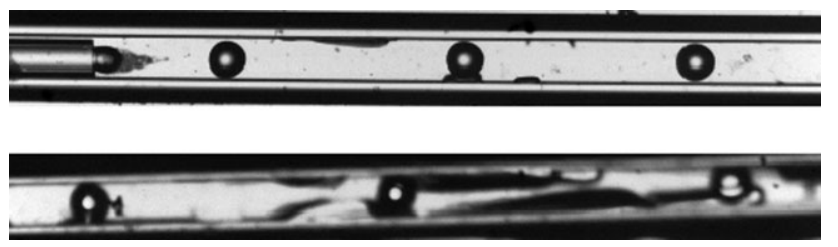

Figure 9. Optical microscopy picture of EGDA droplets formed in alginate solution: (a) system before the application of UV light and (b) system after $1 \mathrm{~min}$ of UV irradiation. 

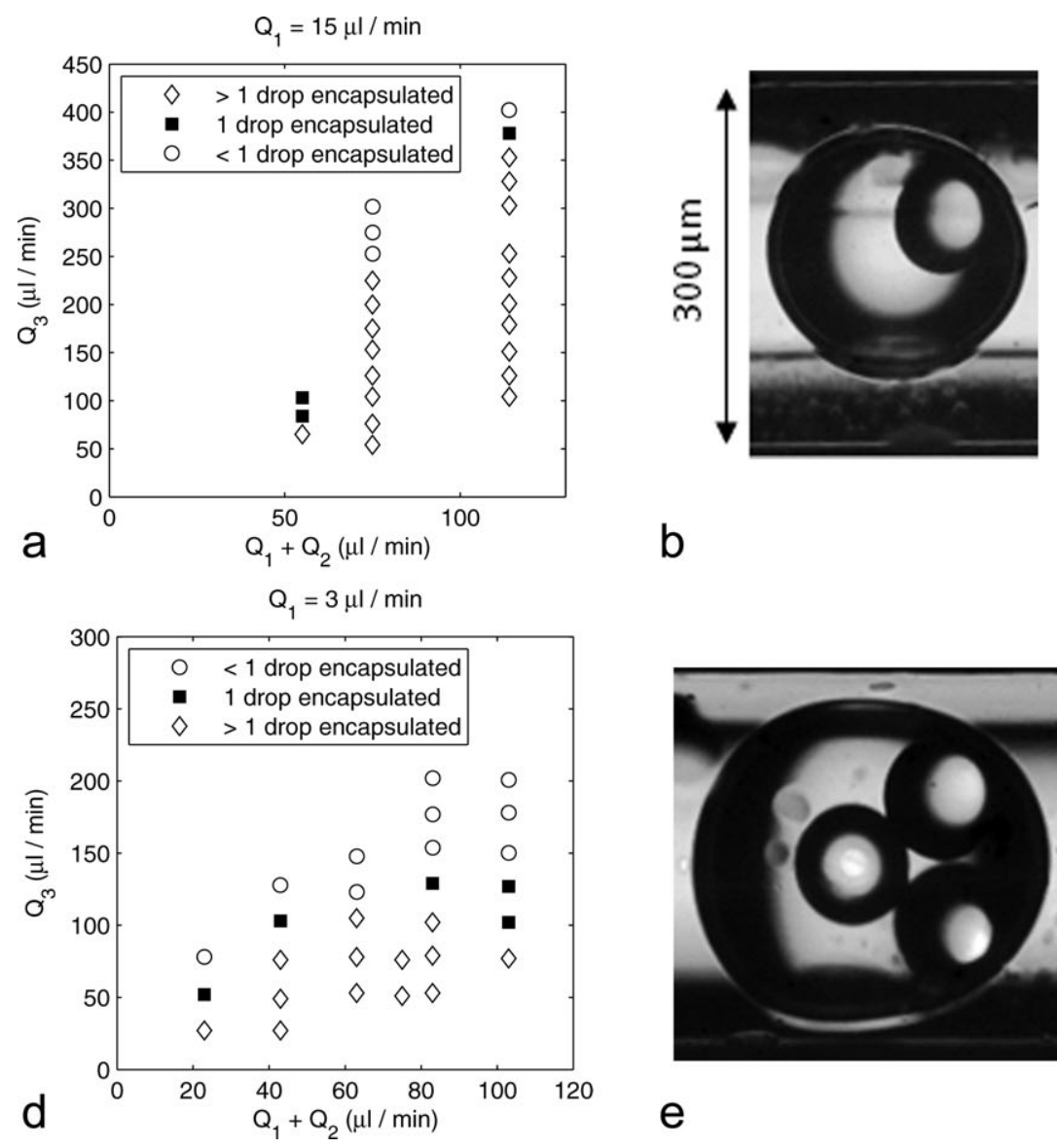

b

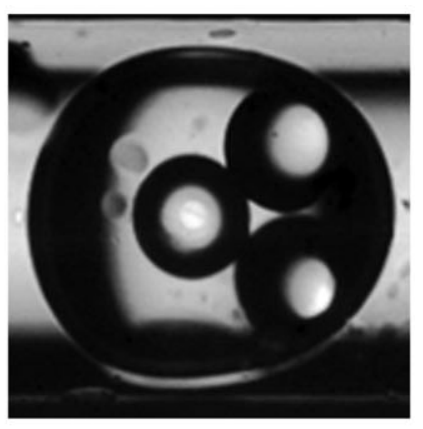

e

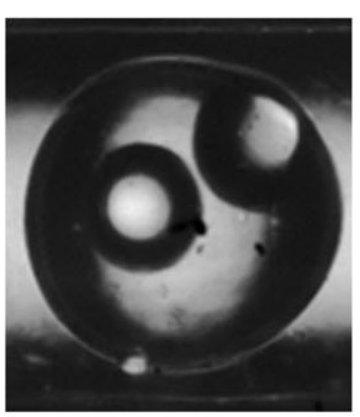

C

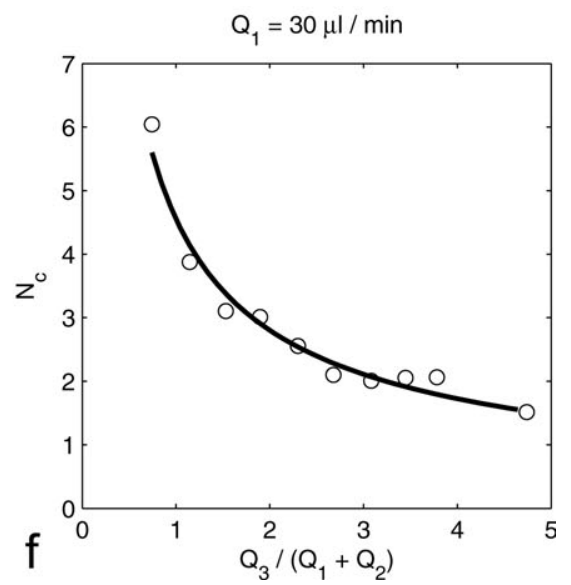

Figure 10. Flow diagrams of the encapsulation stage of the C2 E2 route in the drop only region as functions of internal $\left(Q_{1}+Q_{2}\right)$ and external $\left(Q_{3}\right)$ flow rates for (a) $Q_{1} \quad 15 \mu \mathrm{L} / \mathrm{min}$, (d) $Q_{1} \quad 3 \mu \mathrm{L} / \mathrm{min}$. (b) Capsule with two cores obtained for $Q_{1} \quad 3 \mu \mathrm{L} / \mathrm{min}, Q_{2} \quad 40 \mu \mathrm{L} / \mathrm{min}$, and $Q_{3} \quad 75 \mu \mathrm{L} / \mathrm{min}$. (c) Capsule with a single core obtained for $Q_{1} \quad 3 \mu \mathrm{L} / \mathrm{min}, Q_{2} \quad 40 \mu \mathrm{L} / \mathrm{min}$, and $Q_{3} \quad 100 \mu \mathrm{L} / \mathrm{min}$. (e) Capsule with three cores obtained for $Q_{1} \quad 15 \mu \mathrm{L} / \mathrm{min}, Q_{2} \quad 60 \mu \mathrm{l} / \mathrm{min}$, and $Q_{3} \quad 125 \mu \mathrm{L} / \mathrm{min}$. (f) Variation of the average number of EGDA cores per capsule as a function of the imposed flow ratio for $Q_{1} \quad 30 \mu \mathrm{L} / \mathrm{min}$. The solid curve is the best fit of the data set to a power law and leads to $N_{c} \sim\left[Q_{3} /\left(Q_{1}+Q_{2}\right)\right]^{0.7}$.

salted oleic acid in vials. The minimal residence time of alginate droplets in $0.4 \mathrm{wt} \%$ salted oleic acid required for achieving homogeneous gelation was found to be $1 \mathrm{~min}$, which could be attained in the millifluidic setup by tuning the alginate and salted oleic acid flow rates within the ranges of encapsulation of a single core. About $0.1 \mathrm{wt} \%$ of Pluronic F127 was also added to the alginate solution in order to avoid adsorption of EGDA droplets at the oleic acid alginate interface.

The inline association of the oleic acid mixing stage, the core fabrication stage, and the encapsulation stage is schematized in Figure 7. The encapsulation step is achieved using an outer cap illary glass tube with circular cross section and dimensions (ID $=800 \mu \mathrm{m}$ and $\mathrm{OD}=1 \mathrm{~mm}$ ). The flow downstream from the encapsulation stage is eventually submitted to UV irradiation that triggers the polymerization of the EGDA core. The dynamic flow diagrams of the encapsulation step measured for two different values of the EGDA solution flow rate $Q_{1}$ are shown in Figure 10(a, d). Figure 10(f) displays the dependence of the average number of EGDA cores per alginate droplet $N_{\mathrm{C}}$ on the flow rate ratio. These diagrams allow for the determination of the experimental conditions required for encapsulating a single EGDA core inside each alginate droplet. Finally, Figure 11 displays the variations of the outer diameter of the core shell particles $2 b$ versus the flow rate ratio $Q_{3} /\left(Q_{1}+Q_{2}\right)$. Again, their best power law fit, $2 b \propto\left(Q_{3} /\left(Q_{1}+Q_{2}\right)\right)^{-0.17}$, is in good agreement with the empirical law evidenced in Ref. 24.

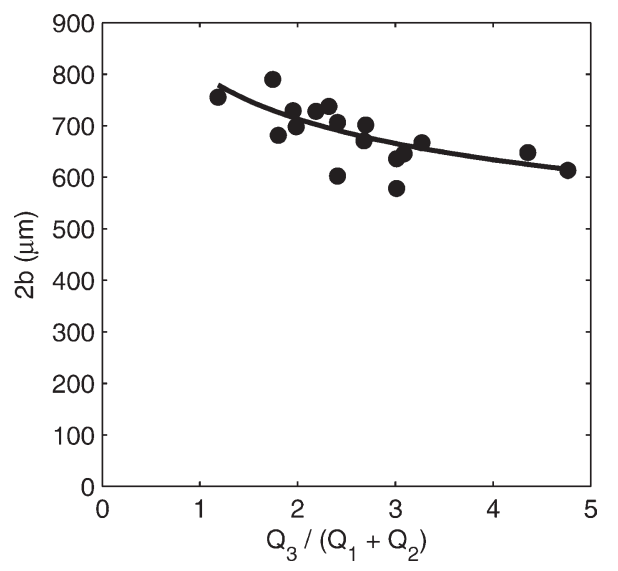

Figure 11. Evolution of the outer diameter of the core shell particles as a function of the flow rate ratio. The continuous line is the best fit of the data to a power law and leads to $2 b \sim\left[Q_{3} /\left(Q_{1}+Q_{2}\right)\right]^{0.17}$. 


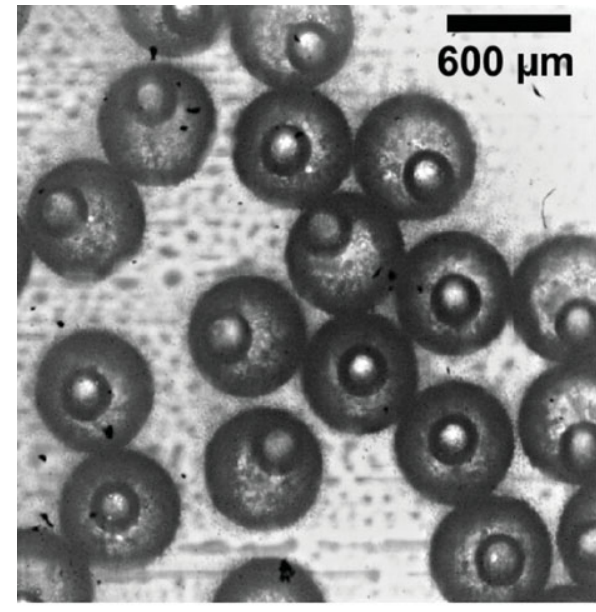

a

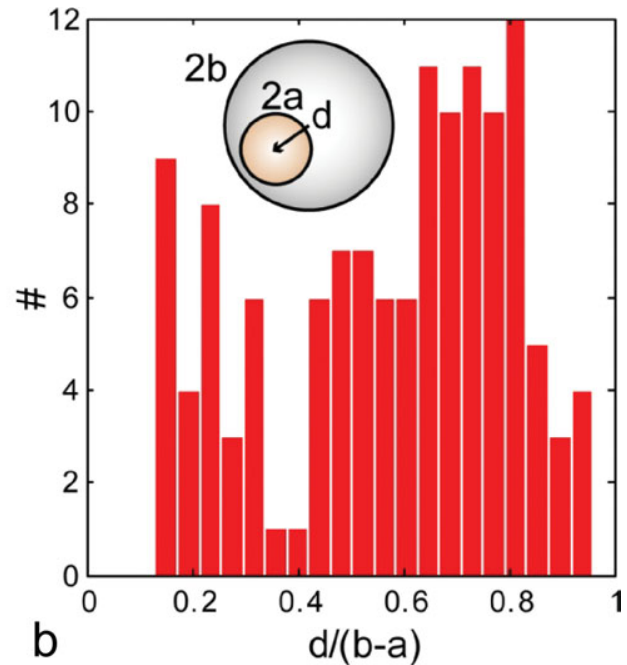

Figure 12. (a) Optical microscopy picture of the core shell particles whose core is made of an initially liquid polymer, which is in line polymerized through UV irradiation and whose shell is made of another polymer, which is polymerized through in line complexation. (b) Histogram of the observed dimensionless off centering of the core within the shell. [Color figure can be viewed in the online issue, which is available at wileyonlinelibrary.com.]

Considering only the capsules containing a single core, capsules having an external diameter ranging from 450 to $750 \mu \mathrm{m}$ and containing a core with diameter ranging from 180 to $380 \mu \mathrm{m}$ could be obtained, corresponding to values of the shell thickness to core radius ratio $(b-a) / a$ ranging from 0.2 to 3 . A picture of the resonators obtained in experimental conditions corre sponding to a weak core confinement is shown in Figure 12(a). A noticeable amount of capsules displays off centered cores. A histogram of the ratio between the measured distance between the core center and the capsule center $d$ and the shell average thickness $b-a$ [see inset of Figure 12(b)] is shown in Figure 12(b). It evidences that a pronounced core off centering is the mostly encountered configuration, which should have important consequences on the acoustic performances of a suspension of such core shell resonators.

\section{Comparison Between the Two Routes}

Both explored routes have resulted in two different types of hard core soft shell particles. Let us compare their characteristic features. Although Route 1 allows using solid cores, it requires density matching for injecting them into the millifluidic circuit. Conversely, Route 2 is less dependent on gravity, but it requires the solidification of the core, which constitutes a constraint for the selection of the material constituting the core. Although in Route 1, the diameter of the solid core is determined from the start; in Route 2, it can be tuned by adjusting the flow rates. Moreover, in Route 1, the injection of solid cores imposes a minimum value of the inner diameter of the capillary through which the cores are injected in order to avoid blocking prob lems. This reduces the number of free parameters controlling the encapsulation process in Route 1. Conversely, in Route 2, the shell thickness to core radius ratio could be tuned from 0.2 to 3 by varying the flow rates.

\section{CONCLUSION}

The formation of mechanical resonators with submillimeter size is a required step toward the realization of 3D LRAM working in the ultrasonic frequency range. We have proposed and imple mented two routes to obtain solid core solid shell particles with submillimeter characteristic size. By contrast with previously developed processes of fabrication of mechanical resonators based on machining and hand made approaches, the routes we propose are based on millifluidic assisted self assembling. Our detailed presentation of the implemented millifluidic processes encompasses original solutions to several practical issues that are the injection of non Brownian solid particles into micro channels, the control of a two phase co flow with simultaneous polymerization, and the diffusion based control of in line poly merization. These processes should be significant milestones toward the millifluidic based synthesis of LRAM. We are now currently working on both the acoustic characterization of the formed objects and on the improvements of their characteristics in terms of dimensions and density contrast.

\section{ACKNOWLEDGMENTS}

We thank Region Aquitaine, CNRS, the SAMM project of the GIS "Advanced Materials in Aquitaine" network, and ANR (grant METAKOUSTIK number ANR2011 BS0902102) for funding and support.

\section{REFERENCES}

1. Veselago, V. G. Sov. Phys. Usp. 1968, 10, 509.

2. Shelby, R. A.; Smith, D. R.; Schultz, S. Science 2001, 292, 77.

3. Engheta, N.; Ziolkowski, R. W. Metamaterials: Physics and Engineering Explorations; Wiley: New York, 2006.

4. Li, J.; Fung, K. H.; Liu, Z. Y.; Sheng, P.; Chan, C. T. In Physics of Refraction and Negative Index Materials; Krowe, C. M., Zhang, Y., Eds.; Springer: New York, 2007; pp 183216.

5. Lu, M. H.; Zhang, C.; Feng, L.; Zhao, J.; Chen, Y. F.; Mao, Y. W.; Zi, J.; Zhu, Y. Y.; Zhu, S. N.; Ming, N. B. Nat. Mater. 2007, 6, 744. 
6. Zhang, S.; Yin, L.; Fang, N. Phys. Rev. Lett. 2009, 102, 194301.

7. Zhang, S.; Xia, C.; Fang, N. Phys. Rev. Lett. 2011, 106, 024301 .

8. Li, J.; Fok, L.; Yin, X.; Bartal, G.; Zhang, X. Nat. Mater. 2009, 8, 931.

9. Liu, Z.; Zhang, X.; Mao, Y.; Zhu, Y. Y.; Yang, Z.; Chan, C. T.; Sheng, P. Science 2000, 289, 1734.

10. Yang, Z.; Mei, J.; Yang, M.; Chan, N. H.; Sheng, P. Phys. Rev. Lett. 2008, 101, 04301.

11. Fang, N.; Xi, D.; Xu, J.; Ambati, M.; Srituravanich, W.; Sun, C.; Zhang, X. Nat. Mater. 2006, 5, 452.

12. Lee, S. H.; Park, C. M.; Seo, Y. M.; Wang, Z. G.; Kim, C. K. Phys. Rev. Lett. 2010, 104, 054301.

13. Park, C. M.; Park, J. J.; Lee, S. H.; Seo, Y. M.; Kim, C. K.; Lee, S. H. Phys. Rev. Lett. 2011, 107, 194301.

14. Lu, M. H.; Feng, L.; Cheng, Y. F. Mater. Today 2009, 12, 34.

15. Liu, Z.; Chan, C. T.; Sheng, P. Phys. Rev. B 2005, 71, 014103.
16. Guillot, P.; Colin, A.; Utada, A. S.; Ajdari, A. Phys. Rev. Lett. 2007, 99, 104502.

17. Utada, A. S.; Lorenceau, E.; Link, D. R.; Kaplan, P. D.; Stone, H. A.; Weitz, D. A. Science 2005, 308, 537.

18. Kumacheva, E.; Garstescki, P. Microfluidic Reactors for Polymer Particles; Wiley: United Kingdom, 2011.

19. Ji, X. H.; Cheng, W.; Guo, F.; Liu, M.; Guo, S. S.; He, Z. K.; Zhao, X. Z. Lab Chip 2011, 11, 2561.

20. Kim, S. H.; Jeon, S. J.; Yang, S. M. J. Am. Chem. Soc. 2008, 130, 6040 .

21. Kumachev, A.; Greener, J.; Tumarkin, E.; Eiser, E.; Zandstra, P. W.; Kumacheva, E. Biomaterials 2011, 32, 1477.

22. Seiffert, S.; Thiele, J.; Abate, A. R.; Weitz, D. A. J. Am. Chem. Soc. 2010, 132, 6606.

23. Abate, R. A.; Chen, C. H.; Agresti, J. J.; Weitz, D. A. Lab Chip 2009, 9, 2628.

24. Serra, C.; Beton, N.; Bouquey, M.; Prat, L.; Hadziioannou, G. Langmuir 2007, 23, 7745.

25. Kim, C.; Chung, S.; Kim, Y. E.; Lee, K. S.; Lee, S. H.; Oh, K. W.; Kang, J. Y. Lab Chip 2011, 11, 246. 